\title{
Robust Fuzzy Control for Stabilization of a Quadrotor
}

\author{
A. Rabhi, M. Chadli and C. Pegard
}

\begin{abstract}
In this paper, we propose an algorithm based on fuzzy control to ensure the stability of the quadrotor. After giving the nonlinear model of the robot, its representation by a Takagi-Sugeno (T-S) fuzzy model is first discussed. Next, a fuzzy controller are synthesized which guarantee desired control performances. The given controller is designed using numerical tools (Linear Matrix Inequalities-LMI). The simulation results show effectiveness and robustness of the proposed method.
\end{abstract}

Key words - Quadrator, nonlinear model, fuzzy control, Linear Matrix Inequalities (LMI).

\section{INTRODUCTION}

$\mathrm{M}$ any research groups are interesting in Unmanned Aerial Vehicles (UAVs). In fact, they provides tremendous advantages and is eligible for applications like rescue and research, remote inspection, surveillance, military applications, therefore saving human pilots from dangerous flight conditions.

Current UAV designs are built from scratch and use customized airframes and electronics, which dramatically increase cost. Another aspect of current designs that limits UAV adoption, especially in the field of academic research, is the lack of access.

When using UAVs for research it is advantageous to have low-level access to both autopilot source code and hardware schematics. There is a large variety of shape for UAV, planes, helicopters... Many works in the literature deal with development and the implementation of nonlinear controllers for quadrotors. Linear control systems such as proportional derivative controllers or linear quadratic regulators are widely used to enhance the stability properties of equilibrium [1].

In this paper, we present an algorithm based on fuzzy control to improve the stability of the robot. After giving his nonlinear model, its representation by the T-S fuzzy model is discussed. Next, the fuzzy controller is developed [12-25]. The closed loop stability conditions of a quadrotor are given in terms of Linear Matrix Inequality (LMI) problem which can be solved very efficiently using the convex optimization techniques. The numerical simulation of the vehicle handling with and without the developed controller has been

A. Rabhi, M. Chadli and C. Pegard are with "Laboratoire Modélisation, Information et Systèmes", UPJV-MIS (EA 4290), 7, Rue Moulin Neuf, 80000 Amiens, France, e-mail: abdelhamid.rabhi@u-picardie.fr carried out to demonstrate the effectiveness of the proposed algorithm in terms of improving the robot stability.

\section{DYNAMIC MODELING}

A quadrotor helicopter consists of four rotors that are mounted at the end of two perpendicular axes as depicted in Fig 1.

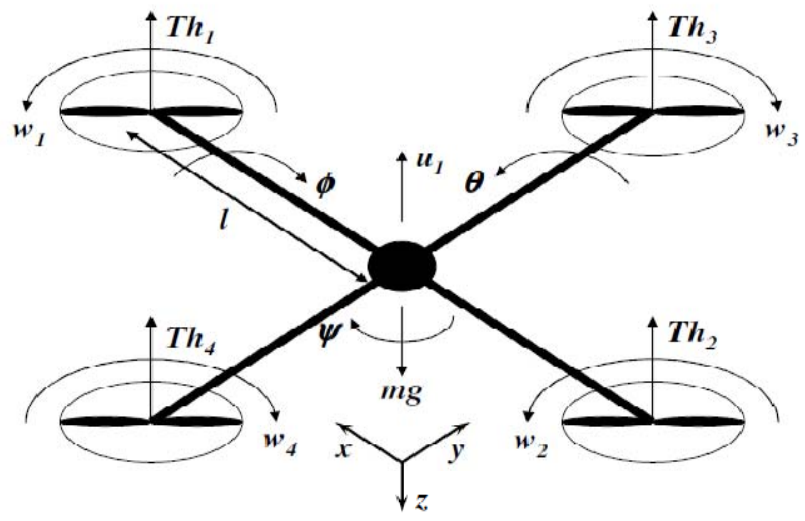

Fig. 1. Quadrotor model

In the literature, many studies deal with dynamic modeling and control for quadrotor [5][6]. There are complex and nonlinear systems. The complete models are difficult to use in control applications. The most part of applications deal with simplified and partial models [7].

In our case, we are interesting to stabilize a robot in air. That is used generally in the phase landing and of take-off of robot. For that we use the model described as follows:

$$
\begin{aligned}
& \ddot{\phi}=\frac{1}{I_{x}}\left\{\dot{\theta} \dot{\psi}\left(I_{y}-I_{z}\right)-K_{f a x} \dot{\phi}^{2}-J_{r} \bar{\Omega} \dot{\theta}+d U_{2}\right\} \\
& \ddot{\theta}=\frac{1}{I_{y}}\left\{\dot{\phi} \dot{\psi}\left(I_{z}-I_{x}\right)-K_{f a y} \dot{\theta}^{2}-J_{r} \bar{\Omega} \dot{\phi}+d U_{3}\right\} \\
& \ddot{\psi}=\frac{1}{I_{z}}\left\{\dot{\theta} \dot{\phi}\left(I_{x}-I_{y}\right)-K_{f a z} \dot{\psi}^{2}+K_{d} U_{4}\right\}
\end{aligned}
$$

Where $U_{i}$ are the control input of motor, $i=1,2,3,4$ the motor number, and $\bar{\Omega}=\left(\omega_{1}-\omega_{2}+\omega_{3}-\omega_{4}\right)$ 


$$
\left[\begin{array}{l}
U_{1} \\
U_{2} \\
U_{3} \\
U_{4}
\end{array}\right]=\left(\begin{array}{cccc}
K_{p} & K_{p} & K_{p} & K_{p} \\
-K_{p} & 0 & K_{p} & 0 \\
0 & -K_{p} & 0 & K_{p} \\
K_{d} & -K_{d} & K_{d} & K_{d}
\end{array}\right)\left[\begin{array}{c}
\omega_{1}^{2} \\
\omega_{2}^{2} \\
\omega_{3}^{2} \\
\omega_{4}^{2}
\end{array}\right]
$$

For more detailed of this model, readers can see [8].

Table 1: Quadrotor Parameters

\begin{tabular}{|c|c|}
\hline Parameter & Description \\
\hline$\phi$ & Roll angle [rad] \\
\hline$\theta$ & Pitch angle [rad] \\
\hline$\psi$ & Yaw angle [rad] \\
\hline$\omega_{i}$ & Rotor speed (i=1,2,3,4) [rad/s] \\
\hline$I_{x}$ & Moment of inertia along x \\
\hline$I_{y}$ & Moment of inertia along $\mathrm{y}$ \\
\hline$I_{z}$ & Lift coefficient \\
\hline$K_{p}$ & The drag coefficient \\
\hline$K_{d}$ &
\end{tabular}

The model can be written in the form:

$$
\dot{x}(t)=A(x) x(t)+B(x) u(t)
$$

where

$$
\begin{aligned}
x & =\left[\begin{array}{llllll}
\theta & \varphi & \psi & \dot{\theta} & \dot{\varphi} & \dot{\psi}
\end{array}\right]^{T}, y=\left[\begin{array}{llll}
\theta & \varphi & \psi
\end{array}\right]^{T}, \\
u & =\left[\begin{array}{llllll}
\omega_{1} & \omega_{2} & \omega_{3} & \omega_{1}{ }^{2} & \omega_{2}{ }^{2} & \omega_{3}{ }^{2}
\end{array}\right]^{T}
\end{aligned}
$$$$
A(x)=\left[\begin{array}{cccccc}
0 & 0 & 0 & 1 & 0 & 0 \\
0 & 0 & 0 & 0 & 1 & 0 \\
0 & 0 & 0 & 0 & 0 & 1 \\
0 & 0 & 0 & \frac{-K_{f a x}}{I_{x}} x_{4} & 0 & \frac{\left(I_{y}-I_{z}\right)}{I_{x}} x_{5} \\
0 & 0 & 0 & 0 & \frac{-K_{f a y}}{I_{y}} x_{5} & \frac{\left(I_{z}-I_{x}\right)}{I_{y}} x_{5} \\
0 & 0 & 0 & \frac{\left(I_{x}-I_{y}\right)}{I_{z}} x_{5} & 0 & \frac{-K_{f a x}}{I_{y}} x_{6}
\end{array}\right]
$$

$$
B(x)=\left[\begin{array}{cccccccc}
0 & 0 & 0 & 0 & 0 & 0 & 0 & 0 \\
0 & 0 & 0 & 0 & 0 & 0 & 0 & 0 \\
0 & 0 & 0 & 0 & 0 & 0 & 0 & 0 \\
-J_{r} x_{5} & J_{r} x_{5} & -J_{r} x_{5} & J_{r} x_{5} & -d K_{p} & 0 & d K_{p} & 0 \\
-J_{r} x_{4} & J_{r} x_{4} & -J_{r} x_{4} & J_{r} x_{4} & 0 & -d K_{p} & 0 & d K_{p} \\
0 & 0 & 0 & 0 & K_{d}{ }^{2} & -K_{d}{ }^{2} & K_{d}{ }^{2} & K_{d}{ }^{2}
\end{array}\right]
$$

\section{FUZZY REPRESENTATION}

In this work, the Takagi-Sugeno (TS) approach is used [1225].

\section{A. TS Fuzzy model}

The angular speed are assumed varying in the operating range:

$$
\varphi_{\text {min }}<\hat{\dot{\varphi}}<\varphi_{\text {max }}, \theta_{\text {min }}<\hat{\dot{\theta}}<\theta_{\text {max }}, \psi_{\text {min }}<\hat{\dot{\psi}}<\psi_{\text {max }},
$$

Consequently we can consider the following IF-THEN rules:

If $\hat{\dot{\varphi}}$ is $\mu_{1}^{1}, \hat{\dot{\theta}}$ is $\mu_{2}^{1}$ and $\hat{\dot{\psi}}$ is $\mu_{3}^{1}$ Then $\left\{\begin{array}{l}\dot{x}=A_{1} x+B_{1} u \\ y=C x\end{array}\right.$

If $\hat{\dot{\varphi}}$ is $\mu_{1}^{1}, \hat{\dot{\theta}}$ is $\mu_{2}^{2}$ and $\hat{\dot{\psi}}$ is $\mu_{3}^{1}$ Then $\left\{\begin{array}{l}\dot{x}=A_{2} x+B_{2} u \\ y=C x\end{array}\right.$

If $\hat{\dot{\varphi}}$ is $\mu_{1}^{1}, \hat{\dot{\theta}}$ is $\mu_{2}^{1}$ and $\hat{\dot{\psi}}$ is $\mu_{3}^{2}$ Then $\left\{\begin{array}{l}\dot{x}=A_{3} x+B_{3} u \\ y=C x\end{array}\right.$

If $\hat{\dot{\varphi}}$ is $\mu_{1}^{1}, \hat{\dot{\theta}}$ is $\mu_{2}^{2}$ and $\hat{\dot{\psi}}$ is $\mu_{3}^{2}$ Then $\left\{\begin{array}{l}\dot{x}=A_{4} x+B_{4} u \\ y=C x\end{array}\right.$

If $\hat{\dot{\varphi}}$ is $\mu_{1}^{2}, \hat{\dot{\theta}}$ is $\mu_{2}^{1}$ and $\hat{\dot{\psi}}$ is $\mu_{3}^{1}$ Then $\left\{\begin{array}{l}\dot{x}=A_{5} x+B_{5} u \\ y=C x\end{array}\right.$

If $\hat{\dot{\varphi}}$ is $\mu_{1}^{2}, \hat{\dot{\theta}}$ is $\mu_{2}^{2}$ and $\hat{\dot{\psi}}$ is $\mu_{3}^{1}$ Then $\left\{\begin{array}{l}\dot{x}=A_{6} x+B_{6} u \\ y=C x\end{array}\right.$

If $\hat{\dot{\varphi}}$ is $\mu_{1}^{2}, \hat{\dot{\theta}}$ is $\mu_{2}^{1}$ and $\hat{\dot{\psi}}$ is $\mu_{3}^{2}$ Then $\left\{\begin{array}{l}\dot{x}=A_{7} x+B_{7} u \\ y=C x\end{array}\right.$

If $\hat{\dot{\varphi}}$ is $\mu_{1}^{2}, \hat{\dot{\theta}}$ is $\mu_{2}^{2}$ and $\hat{\dot{\psi}}$ is $\mu_{3}^{2}$ Then $\left\{\begin{array}{l}\dot{x}=A_{8} x+B_{8} u \\ y=C x\end{array}\right.$

Nonlinear model (1) can be then represented by eight local LTI models. The overall T-S fuzzy model for the quadrotors can be written as follows:

$$
\left\{\begin{array}{l}
\dot{x}(t)=\sum_{i=1}^{n=8} h_{i}(x)\left(A_{i} x(t)+B_{i} u(t)\right) \\
y(t)=C x(t)
\end{array}\right.
$$

where

$$
\begin{aligned}
& h_{1}(z(t))=\mu_{1}^{1} * \mu_{2}^{1} * \mu_{3}^{1}, h_{2}(z(t))=\mu_{1}^{1} * \mu_{2}^{2} * \mu_{3}^{1} \\
& h_{3}(z(t))=\mu_{1}^{1} * \mu_{2}^{1} * \mu_{3}^{2}, h_{4}(z(t))=\mu_{1}^{1} * \mu_{2}^{2} * \mu_{3}^{2} \\
& h_{5}(z(t))=\mu_{1}^{2} * \mu_{2}^{1} * \mu_{3}^{1}, h_{6}(z(t))=\mu_{1}^{2} * \mu_{2}^{2} * \mu_{3}^{1} \\
& h_{7}(z(t))=\mu_{1}^{2} * \mu_{2}^{1} * \mu_{3}^{2}, h_{8}(z(t))=\mu_{1}^{2} * \mu_{2}^{2} * \mu_{3}^{2}
\end{aligned}
$$


with

$$
\begin{aligned}
& \mu_{1}^{1}=\frac{-x_{4}+x_{4 \max }}{x_{4 \max }-x_{4 \min }}, \mu_{1}^{2}=\frac{x_{4}-x_{4 \min }}{x_{4 \max }-x_{4 \min }}, \\
& \mu_{2}^{1}=\frac{-x_{5}+x_{5 \max }}{x_{5 \max }-x_{5 \min }}, \mu_{2}^{2}=\frac{x_{5}-x_{5 \min }}{x_{5 \max }-x_{5 \min }} \\
& \mu_{3}^{1}=\frac{-x_{6}+x_{6 \max }}{x_{6 \max }-x_{6 \min }}, \mu_{3}^{2}=\frac{x_{6}-x_{6 \min }}{x_{6 \max }-x_{6 \min }}
\end{aligned}
$$

\section{B. TS Fuzzy controller}

To design a fuzzy controller for the fuzzy model (5), the socalled Parallel Distributed Compensation (PDC) has been employed [12][13].

$$
u(t)=\sum_{i=1}^{8} h_{i}(z) K_{i} x(t)
$$

The closed-loop TS fuzzy model (4) becomes

$$
\left\{\begin{array}{l}
\left.\dot{x}(t)=\sum_{i=1}^{n=8} \sum_{j=1}^{n=8} h_{i}(x) h_{j}(x)\left(A_{i}+B_{i} K_{j}\right) x(t)\right) \\
y(t)=C x(t)
\end{array}\right.
$$

The objective is to design a fuzzy feedback controller for robust stabilization of system (2) in the form (5) where $K_{i}$ is the controller gains to be determined.

In this work, stabilization conditions using state feedback controller of the T-S model (6) are given. Our study is based on fuzzy control given by the following result [15][16].

Theorem 1: Suppose that there exist matrices $Q>0, \Gamma_{i j}$ and $M_{i}$ satisfying the following LMI conditions $\forall(i, j) \in I_{n}^{2}, i \leq j:$

$$
\begin{aligned}
& \Phi_{i i}+\Gamma_{i i}<0 \\
& \Phi_{i j}+\Phi_{j i}+\Gamma_{i j}^{T}+\Gamma_{i j}<0 \\
& \left(\begin{array}{cccc}
\Gamma_{11} & \Gamma_{12} & \cdots & \Gamma_{1 n} \\
\Gamma_{12}^{T} & \Gamma_{22} & \cdots & \Gamma_{2 n} \\
\vdots & \vdots & \ddots & \vdots \\
\Gamma_{1 n}^{T} & \Gamma_{2 n}^{T} & \ldots & \Gamma_{n n}
\end{array}\right)>0
\end{aligned}
$$

with

$$
\Phi_{i j}=Q A_{i}^{T}+A_{i} Q-M_{j}^{T} B_{i}^{T}-B_{i} M_{j}
$$

Then the fuzzy model (6) is globally asymptotically stable via control law $u(t)=-\sum_{i=1}^{n} h_{i}(z(t)) K_{i} x(t)$ with

$$
K_{i}=M_{i} Q^{-1}
$$

Proof: The proof is obtained by considering the Lyapunov function $V(x)=x(t)^{T} Q^{-1} x(t), Q>0$ (see for example [15]).

\section{High Order Sliding Mode Observer (HOSM)}

Since the activation functions $h_{i}$ (.) depend on immeasurable parameters, a technique based on robust differentiator to estimate the angular speed of robot is used. This observer is used in cascade to obtain the fuzzy model.

The Robust Differentiation Estimator is useful for retrieval of the velocities and accelerations. It's proved that it's converge in finite time [9]. This approach allows to avoid the observability problems dealing with inappropriate use of modeling equations.

Consider a smooth dynamics function, $s(x) \in R$. The system containing this variable may be closed by some possibly dynamical discontinuous feedback where the control task may be to keep the output $s(x(t))=0$.

The sliding variable, the $\mathrm{r}^{\text {th }}$ order sliding mode is determined by: $s=\dot{s}=\ddot{s}=\ldots=s^{(r-1)}=0$, forms an r-dimensional condition on the state of dynamic system[10][11].

The HOSM observer estimates the successive derivatives and gives better robust performance than traditional first order sliding mode. HOSM dynamics converge toward the origin of surface coordinates in finite time always that the order of the sliding controller is equal or bigger than the sum of a relative degree of the plant and the actuator.

Let input signal $f(t)$ be measurable function and it consist of base signal having a derivative with Lipschitz's constant $C>0$. In order to differentiate the input signal, consider the auxiliary equation $\dot{x}=u$ and the following sliding surface which represent the difference between $x$ and $f(t)$ :

$$
s=x-f(t)
$$

By differentiating $s$, it leads to the following relationship

$$
\dot{s}=u-\dot{f}(t)
$$

The super twisting algorithm defines the control law $u$ as $u=u_{1}-\lambda|s|^{\frac{1}{2}} \operatorname{sgn}(s) \quad$ with $\quad \dot{u}_{1}=w \operatorname{sgn}(s) \quad$ and $w>0, \lambda>0$ are positive constants. Here $u$ is the output of the differentiator. 
The corresponding sufficient condition for finite time convergence is [9]:

$$
\begin{aligned}
& w>C \\
& \lambda^{2} \geq 4 C \frac{w+C}{w-C}
\end{aligned}
$$

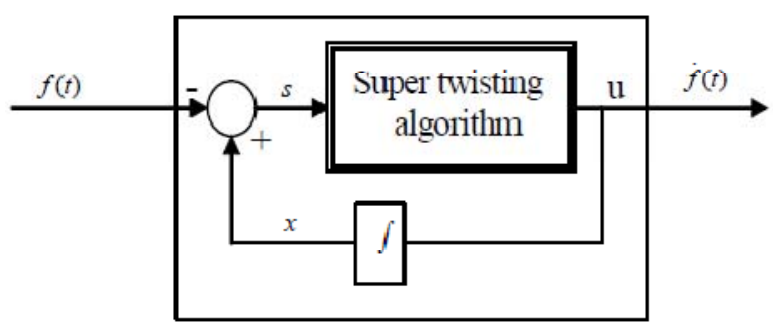

Fig. 2. Structure of the differentiator

\section{SIMULATION RESULTS}

The proposed TS model and designed controller are tested by simulations. This section shows efficiency of the designed controller through computer simulations. Software packages (MATLAB, SIMULINK, LMI Control Toolbox) are used for the simulations.

The simulation results based on the proposed design algorithm are shown in Figure 3. The simulation results show that the fuzzy controller is able to stabilize the used quadrotor.

\section{CONCLUSION}

This paper proposes the Takagi-Sugeno fuzzy approach and a fuzzy controller scheme for the stabilization of a quadrotor system. The nonlinear model is first discussed and then represented by a T-S fuzzy model. We have designed a state feedback controller via inear matrix inequalities approach. Simulation results are carried out to show that the designed controller ensures the global stability of the closed-loop system.

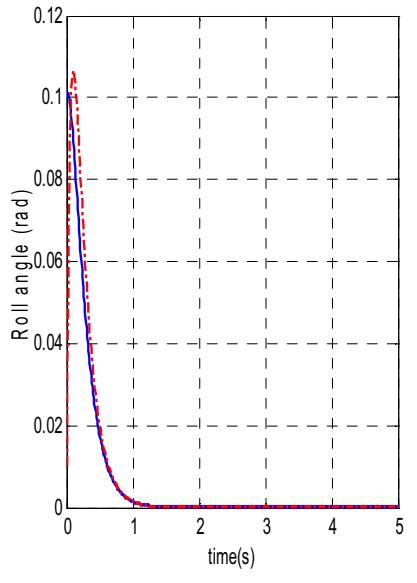

(a)

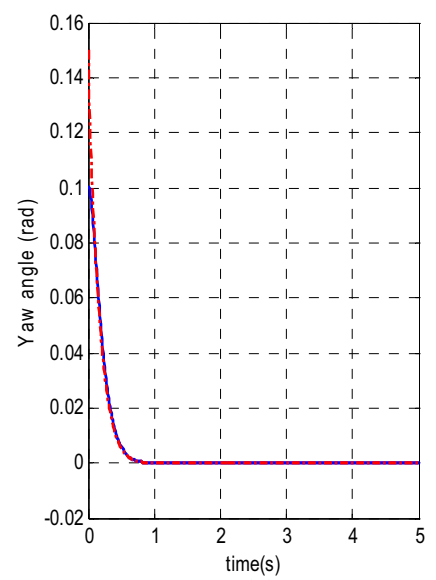

(c)

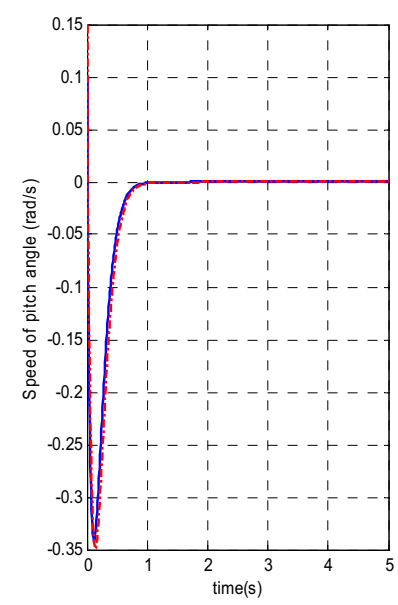

(e)

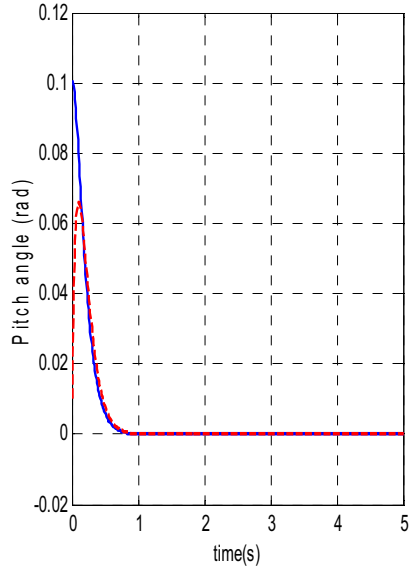

(b)

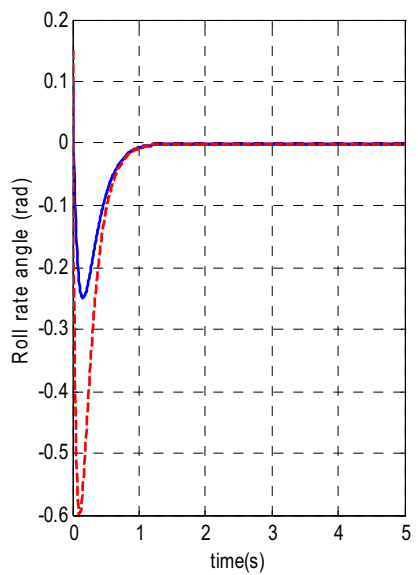

(d)

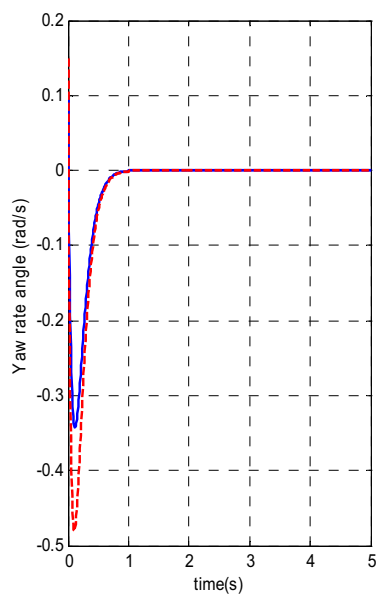

(f)
Figure 3: Simulation results of the Fuzzy controller 


\section{REFERENCES}

[1] M. Valenti, B. Bethke, G. Fiore, and J. How, "Indoor multivehicle flight testbed for fault detection, indoor multi-vehicle flight testbed for fault detection, isolation, and recovery," in Proceedings of the AIAA Guidance, Navigation and Control Conference, 2006.

[2] P. Castillo, R. Lozano and A. Dzul, "Modelling and Control of Mini-Flying Machines", Springer-Verlag in Advances in Industrial Control, July 2005. ISBN: 1-85233-957-8

[3] Castillo P., Lozano R., Dzul A., "Stabilization of a mini rotorcraft with four rotors". Control Systems Magazine, IEEE Vol. 25, pp.45-55, 2005.

[4] Hamel T. Mahoney r. Lozano r. Et Ostrowski j, 2002. "Dynamic modelling and configuration stabilization for an X4-flyer." In the 15éme IFAC world congress', Barcelona, Spain.

[5] S.Bouabdellah and R.Siegwart, 2005 "Backstepping and sliding mode techniques applied to an indoor micro quadrotor" Proceeding of the 2005 IEEE, ICRA, Barcelona, Spain, April.

[6] A.Mokhtari, N.K.M'sirdi, K.Meghriche and A.Belaidi "feedback linearization and linear observer for a quadrotor unmanned aerial vehicle” Advanced Robotics, Vol.20, No.1, pp. 71-91, 2006.

[7] Bora Erginer and Erdinç Altuğ, "Modeling and PD Control of a Quadrotor VTOL Vehicle" Proceedings of the 2007 IEEE Intelligent Vehicles Symposium Istanbul, Turkey, June 13-15, 2007.

[8] H.Bouadi, M.Bouchoucha, M.Tadjine "sliding mode control based on backstepping approach for an UAV type-quadrotor" International Journal of Applied Mathematics and Computer Sciences, Vol.4, No.1, pp. 12-17, Barcelona; Spain 2007.

[9] Levant, A. Higher-order sliding modes, differentiation and output-feedback control, International Journal of Control, 2003, Vol.76, pp.924-941.

[10] A. Levant, "Robust exact differentiation via sliding mode technique", Automatica, vol. 34(3), 1998, pp 379-384.

[11] A. Rabhi, N.K. M'Sirdi, A. Naamane, B. Jaballah, "Estimation of contact forces and road profile using High-Order Sliding Modes" - Int. J. of Vehicle Autonomous Systems 2010 - Vol. 8, No.1 pp. $23-38$

[12] H.O. Wang, K. Tanaka, and M.F. Griffin, "Parallel Distributed Compensation of Nonlinear Systems by Takagi-Sugeno Fuzzy Model”, Proc. FUZZ-IEEE/IFES'95, 1995, pp. 531-538.

[13] M. Takagi and M. Sugeno, "Fuzzy identification of systems and its application to modelling and control", IEEE Trans. on Systems Man and Cybernetics-part C, 15 (1), pp. 116-132, 1985.

[14] K. Tanaka, T. Ikeda, O. Wang, "Fuzzy regulator and fuzzy observer : relaxed stability conditions and LMI based design", IEEE Trans. on Fuzzy Systems, 6 (2), pp. 250-256, 1998.

[15] M. Chadli and A. El Hajjaji, "Observer-based robust fuzzy control of nonlinear systems with parametric uncertaintiescomment on", Fuzzy Sets and Systems Journal, Vol. 157(9), pp. 1276-1281, 2006.

[16] S.C Cao, N. W. Rees, G. Feng. "Hinf control of uncertain fuzzy continuous-time systems", Fuzzy sets and systems, 115, pp. 171-190, 2000.

[17] M. Chadli, D. Maquin, J. Ragot, "Stability analysis and design for continuous-time Takagi-Sugeno control systems", International Journal of Fuzzy Systems, vol. 7, no. 3, 2005

[18] T. M. Guerra, L. Vermeiren, "LMI-based relaxed nonquadratic stabilization conditions for nonlinear systems in the Takagi-Sugeno's form", Automatica, Vo. 40(5), pp. 823829. 2004.

[19] H. J. Lee, J. B. Park, G. Chen, "Robust fuzzy control of nonlinear systems with parametric uncertainties", IEEE Trans. on Fuzzy Systems, 9(2), pp. 369379, 2001.

[20] M. Chadli, A. Elhajjai "Observer-based robust fuzzy control of nonlinear systems with parametric uncertainties-comment on", Fuzzy sets and systems, 2005. to appear.

[21] S. Tong et al. "Fuzzy robust tracking control for uncertain nonlinear systems", International Journal of approximate reasoning (30), pp. 73-90, 2002.

[22] S. Boyd. et al. Linear matrix inequalities in systems and control theory. Philadelphia, PA: SIAM, 1994.

[23] B. S. Chen, C. H. Lee, and Y. C. Chang, " $H^{\infty}$ tracking design of uncertain nonliear SISO systems: Adaptive fuzzy approach," IEEE Trans. Fuzzy Syst. Vol. 4, no. 1, pp. 32-43, Feb. 1996.

[24] M. Oudghiri, M. Chadli and A. El Hajjaji, "Design of Robust Output Hœ Fuzzy Controller Using One-Step Procedure", The 15th IEEE Mediterranean Conference on control and Automation MED'07, Athens, Greece, June 27-29, 2007.

[25] C. Lin, Q. Wang, T. Lee, "Improvement on observer-based Hinf control for T-S fuzzy systems", Automatica, vol. 41, pp 1651-1656, 2005. 